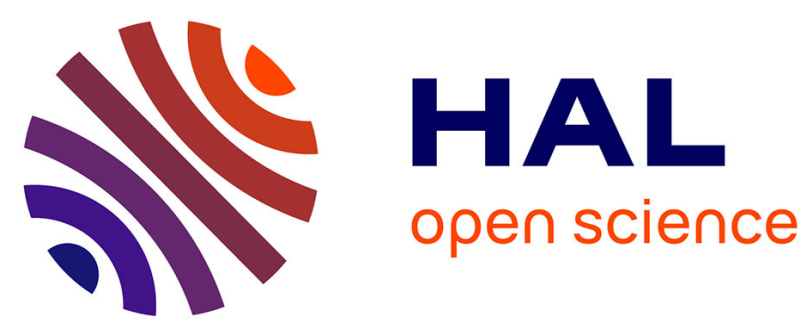

\title{
Biologie des pucerons des céréales dans l'Ouest de la France. III. Action des hyménoptères parasites sur les populations de Sitobion avenae F., Metopolophium dirhodum Wlk. et Rhopalosiphum padi L
}

Jean-Michel Rabasse, Charles-Antoine Dedryver, Augustine Gelle, Jean-Pierre Lafont, Josette Molinari

\section{To cite this version:}

Jean-Michel Rabasse, Charles-Antoine Dedryver, Augustine Gelle, Jean-Pierre Lafont, Josette Molinari. Biologie des pucerons des céréales dans l'Ouest de la France. III. Action des hyménoptères parasites sur les populations de Sitobion avenae F., Metopolophium dirhodum Wlk. et Rhopalosiphum padi L. Agronomie, 1983, 3 (8), pp.779-790. hal-00884571

\section{HAL Id: hal-00884571 \\ https://hal.science/hal-00884571}

Submitted on 1 Jan 1983

HAL is a multi-disciplinary open access archive for the deposit and dissemination of scientific research documents, whether they are published or not. The documents may come from teaching and research institutions in France or abroad, or from public or private research centers.
L'archive ouverte pluridisciplinaire HAL, est destinée au dépôt et à la diffusion de documents scientifiques de niveau recherche, publiés ou non, émanant des établissements d'enseignement et de recherche français ou étrangers, des laboratoires publics ou privés. 


\section{Biologie des pucerons des céréales dans l'Ouest} de la France. III. Action des hyménoptères parasites
sur les populations de Sitobion avenae F., Metopolo-
phium dirhodum Wlk. et Rhopalosiphum padi L.

Jean-Michel RABASSE \& Charles-Antoine DEDRYVER $\left({ }^{*}\right)$

avec la collaboration technique de Augustine Gelle $\left({ }^{*}\right)$, Jean-Pierre LAFONT \& Josette MOLINARI

I.N.R.A., Station de Zoologie et de Lutte biologique, boulevard du Cap, F06602 Antibes.

(*) I.N.R.A., Laboratoire de Recherches de la Chaire de Zoologie de l'E.N.S.A. de Rennes, F35650 Le Rheu

Pendant quatre années, de 1974 à 1977, le parasitisme des pucerons des céréales a été étudié dans diverses localités de Bretagne. Aphidius uzbekistanicus est le principal parasite primaire des 3 pucerons les plus importants; $A$. ervi, $A$. picipes et $A$. matricariae jouent un rôle variable selon les années. Seize espèces d'hyperparasites s'attaquent à ces auxiliaires. Les populations de différents parasites primaires évoluent souvent de façon analogue dans une même parcelle. Un plus grand nombre de parasites sont produits sur le bord des parcelles. Le pourcentage de momies dans les populations de Sitobion avenae est plus élevé sur feuilles que sur épis, par suite de la migration vers le haut de ce puceron. Une préférence au champ d'A. picipes pour $S$. avenae et une préférence, variable dans le temps, d'A. uzbekistanicus pour Metopolophium dirhodum, puis pour $S$. avenae, ont été mises en évidence. Le déséquilibre du sex-ratio à l'éclosion ( $40 \mathrm{p} .100$ de mâles) est confirmé pour $A$. uzbekistanicus, $A$. ervi et $A$. matricariae. Enfin, $A$. uzbekistanicus a rarement une action déterminante dans la limitation des populations aphidiennes sur céréales.

Mots clés additionnels : Aphides, parasitoïdes, Aphidiidae, régulation naturelle des populations. populations of Sitobion avenae $F$., Metopolophium dirhodum $W l k$. and Rhopalosiphum padi $L$.

From 1974 to 1977, the impact of parasites on cereal aphids was studied in several places in Brittany (table 1). Aphidius uzbekistanicus was the main primary parasite of the 3 most important aphids; $A$. ervi, A. picipes and A. matricariae were more or less important according to the year (table 2). Sixteen species of hyperparasites cmerged from the mummies (table 3 ). Populations of different primary parasites often followed similar trends in the same field (fig. 1 and 2). The edges of the fields produced more parasites than the centre (table 4). The percentage of mummies in populations of Sitobion avenae was higher on the leaves than on the ears (table 5), as this aphid migrates upwards. In the fields, we have shown the preference of $A$. picipes for $S$. avenae and of A. uzbekistanicus first for Metopolophium dirhodum, then for S. avenae (table 6). At hatching time, $40 \%$ males were found in populations of A. uzbekistanicus, A. ervi and A. matricariae (table 7). Finally, fig. 3 and 4 show that $A$. uzbekistanicus rarely seemed to act as the main regulation factor of aphids on cereals.

Additional key words : Aphids, parasitoids, Aphidiidae, natural control of populations.

\section{INTRODUCTION}

L'étude des parasites des pucerons des céréales dans l'Ouest de la France se situe au point de rencontre de deux ensembles de travaux :

D'une part, l'observation de l'évolution des populations de pucerons des céréales en Bretagne (DEDRYVER, 1978a et $b ; 1981)$ a montré que, comme dans d'autres régions d'Europe, plusieurs types d'ennemis naturels contribuent à la limitation des populations. Il n'est possible de négliger ni le rôle des parasites, ni celui des champignons pathogènes ou des prédateurs, sans cependant qu'il soit toujours facile d'en séparer les différents effets.

D'autre part, l'analyse de l'action des Hyménoptères parasites de pucerons en Bretagne sur différentes cultures (RABASSE \& BRUNEL, 1977 ; ROBERT et al., 1972 ; ROBERT \& RABASSE, 1977 ; ROBERT, 1979) a montré que chacun des écosystèmes étudiés présente ses caractéristiques propres. 
Sur céréales, nous sommes en présence d'un système complexe comportant plusieurs espèces de pucerons et de parasites. La présente étude, qui a été réalisée dans divers biotopes pendant plusieurs années consécutives, avait pour objectif de préciser l'importance relative des différents parasites primaires et le rôle joué par les plus importants.

\section{MÉTHODES}

\section{A. Prélèvements au champ}

Ce travail constituant une contribution à l'étude des pucerons des céréales et de leurs ennemis en Bretagne, nous ne traiterons que sommairement des conditions culturales, qui ont déjà été précisées par ailleurs (DEDRYVER, 1978a). Les observations ont été poursuivies pendant 4 années (de 1974 à 1977) à Rennes, sur blé d'hiver cv. "Champlein », ainsi que dans des localités très diverses de Bretagne. Dans le tableau 1 sont mentionnés la désignation (A, B...), la localisation des parcelles prospectées, l'année des observations ainsi que le type d'agroécosystème auquel ces parcelles appartiennent (bocager : b ; ouvert : o). A chaque date, un prélèvement a été effectué selon un système complexe, dont les modalités ont été déjà décrites (DEDRYVER, 1978a), comportant un certain nombre de prises élémentaires (une longueur de rang ou un nombre de talles donné) le long de transects. Nous précisons en outre les tailles minimale et maximale des échantillons prélevés chaque année. Le nombre de momies (cocons de nymphose du parasite tissés dans/ou sous la dépouille du puceron-hôte) observées selon cette méthode est très variable au cours de la saison.
Indépendamment des champs suivis régulièrement, des observations complémentaires ont été effectuées occasionnellement dans d'autres régions de France.

\section{B. Traitement des échantillons}

Les momies provenant de chaque échantillon ont été mises en éclosoirs individuels pour obtenir les imagos des différents parasites primaires ou des hyperparasites et les identifier ainsi que l'espèce-hôte. Il n'est généralement pas possible de savoir quel était le parasite primaire lorsque c'est un hyperparasite qui éclôt ; en effet, si les parasites des genres Praon et Ephedrus forment des momies caractéristiques, il n'y a pas de différence marquée entre celles des 4 espèces du genre Aphidius.

Le parasitisme par Hyménoptères est exprimé par le pourcentage de momies pleines par rapport à la population de pucerons sains et de momies. L'image du parasitisme ainsi obtenue est retardée par rapport à son effet sur la population.

\section{LES ESPÈCES DE PARASITES \\ ET LEUR IMPORTANCE RELATIVE}

\section{A. Parasites primaires}

1. Les Aphidiidae du genre Aphidius (tabl. 2)

Nous avons trouvé les espèces suivantes: Aphidius uzbekistanicus Luz., sur Metopolophium dirhodum Wlk., Sitobion avenae F. et Rhopalosiphum padi L., Aphidius ervi Hal. et Aphidius picipes Nees sur $M$. dirhodum et $S$. avenae

\section{TABLEAU}

Localisation des parcelles, dénomination conventionnelle et tailles extrêmes des échantillons prélevés ( $b:$ parcelle bocagère; $o:$ champ ouvert). Localizarion and conventional labelling of fields; minimum and maximum size of samples $(b:$ bocage ; o : open field $)$.

\begin{tabular}{|c|c|c|c|c|c|c|}
\hline \multirow{2}{*}{$\begin{array}{c}\begin{array}{c}\text { Dénomination } \\
\text { conventionnelle }\end{array} \\
\mathrm{A}\end{array}$} & \multirow{2}{*}{$\begin{array}{c}\text { Type de } \\
\text { parcelle }\end{array}$} & \multirow{2}{*}{$\begin{array}{c}\text { Année } \\
1974\end{array}$} & \multirow[b]{2}{*}{ Rennes (35) } & \multicolumn{3}{|c|}{$\begin{array}{c}\text { Nombres minimum et maximum } \\
\text { de talles prélevées } \\
\text { à chaque date }\end{array}$} \\
\hline & & & & & 60 & \\
\hline B & o & 1975 & Le Rheu (35) & 900 & - & 1800 \\
\hline $\mathrm{C}$ & $\mathrm{b}$ & 1975 & Reguigny (56) & 300 & - & 360 \\
\hline $\mathrm{D}$ & b & 1975 & Reguigny (56) & & 300 & \\
\hline $\mathrm{E}$ & o & 1975 & Moreac (56) & & 300 & \\
\hline F & o & 1975 & Moreac (56) & & 300 & \\
\hline $\mathrm{G}$ & o & 1976 & Le Rheu (35) & & 780 & \\
\hline $\mathbf{H}$ & 0 & 1976 & Plonevez-Porzay (29 S) & 180 & - & 360 \\
\hline I & o & 1977 & Le Rheu (35) & 200 & - & 3780 \\
\hline $\mathrm{J}$ & o & 1977 & Le Rheu (35) & 840 & - & 1680 \\
\hline $\mathrm{K}$ & $\mathrm{b}$ & 1977 & La Chapelle Thouarault (35) & 520 & - & 1040 \\
\hline $\mathrm{L}$ & $\mathrm{b}$ & 1977 & La Chapelle Thouarault (35) & 320 & - & 1760 \\
\hline
\end{tabular}


TABLEAU 2

Importance relative des Aphidius parasites primaires de Sitobion avenae $F$. et Metopolophium dirhodum Wlk. sur blé en Bretagne. Pourcentage des momies de ces 2 aphides ayant donné naissance à un parasite de chaque espèce dans les différents biotopes.

Relative abundance of the different Aphidius parasites of Sitobion avenae $F$. and Metopolophium dirhodum Wlk. on wheat in Brittany. Percentage of the mummies of these 2 aphids from which a parasite of each species emerged in several ecosystems.

\begin{tabular}{|c|c|c|c|c|}
\hline \multirow{2}{*}{$\begin{array}{l}\text { Désignation } \\
\text { conventionnelle } \\
\text { des parcelles }\end{array}$} & \multicolumn{3}{|c|}{ Pourcentage de momies } & \multirow{2}{*}{$\begin{array}{l}\text { Effectif total } \\
\text { de momies }\end{array}$} \\
\hline & A. uzbekistanicus & A. ervi & A. picipes & \\
\hline B o 75 & 80 & 3 & 17 & 314 \\
\hline $\mathrm{C}$ b 75 & 79 & 1 & 20 & 355 \\
\hline D b 75 & 74 & $<1$ & 26 & 242 \\
\hline E o 75 & 88 & 2 & 10 & 89 \\
\hline F o 75 & 80 & 14 & 6 & 55 \\
\hline G o 76 & 60 & 40 & - & 58 \\
\hline $\mathrm{H}$ o 76 & 100 & - & - & 133 \\
\hline I o 77 & 87 & 13 & - & 597 \\
\hline J $\circ 77$ & 78 & 22 & - & 372 \\
\hline K b 77 & 89 & 11 & - & 248 \\
\hline L b 77 & 92 & 8 & - & 441 \\
\hline
\end{tabular}

et enfin Aphidius matricariae Hal. sur $R$. padi. L'identification de ces espèces pose actuellement des problèmes délicats qui ont été débattus par ailleurs (RABASSE, 1982).

A. uzbekistanicus a été trouvé régulièrement dans toutes les parcelles sur les 3 aphides chaque fois que ceux-ci étaient présents. C'est de loin le parasite dominant des pucerons des céréales : 80 p. 100 des parasites primaires qui éclosent des momies de $S$. avenae et de $M$. dirhodum appartiennent à cette espèce (tabl. 2). Autant que l'on sache actuellement, ce parasite est lié aux aphides des graminées appartenant aux genres déjà mentionnés, ainsi qu'au genre Schizaphis (STARY, 1973 ; STARY et al., 1973). C'est uniquement cette espèce que nous prendrons en considération plus loin pour apprécier l'action du parasitisme sur les pucerons des céréales.

A. ervi a été observé chaque fois, sauf à Plonevez-Porzay (H), en 1976, où $R$. padi, qu'il ne parasite pas, était le seul puceron abondant.

A. picipes est obtenu dans toutes les parcelles en 1975, ainsi qu'en 1974, mais pas les 2 années suivantes. Ces 2 espèces sont largement polyphages sur d'autres aphides importants des cultures (appartenant notamment aux genres Acyrthosiphon, Macrosiphum...). Le rôle qu'elles jouent sur céréales est certainement très variable en fonction des caractéristiques des populations aphidiennes dans l'ensemble de l'écosystème au cours d'une année donnée.

$A$. matricariae n'est pas toujours présent sur $R$. padi : il a été rencontré seulement en 1975 dans les parcelles B, D, E et dans les 2 champs suivis en 1976, mais il faut souligner que les populations de $R$. padi sont souvent très faibles (sauf à Plonevez-Porzay, en 1976). Ce parasite est considéré comme largement polyphage, notamment sur les aphides appartenant aux genres Brachycaudus, Myzus, Rhopalosiphum (MACKAUER \& STARY, 1967).

\section{Autres Aphidiidae}

Trois espèces appartenant à des genres différents se comportent comme des parasites occasionnels.

Vingt-trois individus de Praon volucre Hal. ont été trouvés dans les champs de blé sur $S$. avenae en 6 occasions aux mois de juin et juillet, ainsi qu'un mâle sur $M$. dirhodum. Ce même parasite a été trouvé sur $R$. padi lors d'essais cn serre.
Ephedrus plagiator Nees n'a été trouvé que sur $S$. avenae à Rennes (11 individus récoltés au mois de juillet). Comme nous le verrons plus loin, des momies caractéristiques des genres Praon et Ephedrus ont également été trouvées hyperparasitées; $P$. volucre et $E$. plagiator n'en sont pas moins des parasites occasionnels des pucerons des céréales en Bretagne.

Notons que l'absence de Toxares deltiger Hal. de nos relevés pourrait s'expliquer, selon POWELL (1980), par le fait que les momies de ce parasite ne sont pas formées sur le végétal.

Nous n'avons trouvé enfin qu'un mâle de Diaeretiella rapae M'Int., parasite typique de Brevicoryne brassicae L., en culture de blé sur $M$. dirhodum, le 9 juin 1976 (G).

\section{Aphelinidae}

Les Aphelinidés sont très exceptionnels dans nos prélèvements. Nous n'avons obtenu que 2 femelles d'Aphelinus abdominalis Dalm. récoltées à Rennes, l'une sur $S$. avenae le 8 juin 1977, l'autre sur $M$. dirhodum le 19 mai 1978. Ce parasite considéré comme lié aux genres Macrosiphum et Acyrthosiphon, avait déjà été obtenu de Sitobion equiseti Holm. (KALINA \& STARY, 1976) et élevé sur S. avenae et $R$. padi par Michel (1969).

Notons qu'une autre espèce, $A$. asychis Wlk., a été élevée sur les 3 espèces d'aphides qui nous intéressent (MicHEL, 1970; MONADJEMI, 1972; RAUTAPAA, 1972) et introduite aux Etats-Unis pour lutter contre Schizaphis graminum Rond. (JACKSON \& EIKENBARY, 1971), mais la littérature n'apporte aucune précision sur son abondance, ni même sur sa présence chez ces aphides dans les champs de céréales d'Europe.

\section{B. Hyperparasites (tabl. 3)}

\section{Ceraphronoidea - Megaspilidae - Dendrocerus}

Les Dendrocerus sont ectoparasites des Aphidiidés contenus dans les momies.

Dendrocerus (Macrostigma) carpenteri Curt. est de loin le mégaspilidé le plus abondant. Cette espèce est connue en Tchécoslovaquie (STARY, 1977) sur les 3 pucerons du blé. En 1975,1976 et 1977 , nous avons trouvé $D$. carpenteri pour la $1^{\text {ire }}$ fois dans des momies récoltées fin mai-début juin. 
TABLEAU 3

Inventaire et importance relative des hyperparasites de Sitobion avenae $F$. (S.a.), Metopolophium dirhodum Wlk. (M.d.) et Rhopalosiphum padi $L$. (R.p.) sur blé en Bretagne, Aphidius spp. étant parasites primaires.

Relative abundance of the hyperparasites of Sitobion avenae $F$. (S.a.), Metopolophium dirhodum Wlk. (M.d.) and Rhopalosiphum padi $L$. (R.p.) on wheat in Brittany, with Aphidius spp. as the primary parasites.

\begin{tabular}{|c|c|c|c|c|c|c|c|c|c|}
\hline & \multicolumn{8}{|c|}{ Nombre d'individus éclos } & \multirow{3}{*}{$\begin{array}{l}\text { Désignation conventionnelle } \\
\text { des parcelles }\end{array}$} \\
\hline & \multicolumn{3}{|c|}{ S.a. } & \multicolumn{3}{|c|}{ M.d. } & \multicolumn{2}{|c|}{ R.p. } & \\
\hline & $q$ & $?$ & $\delta$ & $q$ & $?$ & $\delta$ & $q$ & $\delta$ & \\
\hline $\begin{array}{l}\text { - Ceraphronoidea - Megaspilidae } \\
\text { Dendrocerus (Macrostigma) } \\
\text { carpenteri Curt. }\end{array}$ & 130 & 6 & 105 & 81 & 1 & 72 & 3 & 3 & Toutes parcelles \\
\hline $\begin{array}{l}\text { D. (M.) aphidum Rondani } \\
\text { D. (M.) rectangularis Kieff. } \\
\text { D. (Atritomellus) laticeps } \\
\text { Hedicke }\end{array}$ & $\begin{array}{r}13 \\
1\end{array}$ & & $\begin{array}{l}9 \\
4\end{array}$ & $\begin{array}{l}5 \\
3 \\
2\end{array}$ & & $\begin{array}{l}2 \\
3\end{array}$ & $\begin{array}{l}2 \\
2\end{array}$ & & $\begin{array}{l}\text { Toutes parcelles sauf B.D.E.F. } \\
\text { B.C.D.H.L. } \\
\text { H }\end{array}$ \\
\hline $\begin{array}{l}\text { - Cynipoidea - Cynipidae: } \\
\text { Phaenoglyphis villosa Htg. } \\
\text { P. xanthochroa Forst. } \\
\text { Alloxysta victrix Westw. } \\
\text { A. macrophadna Htg. } \\
\text { A. castanea Kieff. }\end{array}$ & $\begin{array}{l}38 \\
13\end{array}$ & & $\begin{array}{l}32 \\
11\end{array}$ & $\begin{array}{r}35 \\
1 \\
67\end{array}$ & & $\begin{array}{r}40 \\
31 \\
2 \\
2\end{array}$ & $\begin{array}{r}36 \\
4\end{array}$ & $\begin{array}{r}29 \\
5\end{array}$ & $\begin{array}{l}\text { Toutes parcelles } \\
\text { C } \\
\text { Toutes parcelles } \\
\text { B.D. } \\
\text { D }\end{array}$ \\
\hline $\begin{array}{l}\text { - Chalcidoidea - Pteromalidae: } \\
\text { Asaphes vulgaris Wlk. } \\
\text { A. suspensus Nees } \\
\text { Pachyneuron solitarium Htg. } \\
\text { P. aphidis Bouché } \\
\text { P. concolor Först. } \\
\text { Coruna clavata Wlk. }\end{array}$ & $\begin{array}{r}31 \\
10 \\
7 \\
1\end{array}$ & 2 & $\begin{array}{r}27 \\
7 \\
5\end{array}$ & $\begin{array}{r}61 \\
5 \\
8 \\
1 \\
4\end{array}$ & & $\begin{array}{r}45 \\
8 \\
3\end{array}$ & $\begin{array}{l}8 \\
1\end{array}$ & $\begin{array}{r}25 \\
3\end{array}$ & $\begin{array}{l}\text { Toutes parcelles sauf E.F.J. } \\
\text { Toutes parcelles sauf A.F.G. } \\
\text { B.C.D.G.H.L. } \\
\text { G.L. } \\
\text { C } \\
\text { C.D. }\end{array}$ \\
\hline
\end{tabular}

Dans l'ensemble des récoltes, nous avons trouvé 54 p. 100 de femelles, valeur à rapprocher des 63 p. 100 obtenus en élevage par BENNETT \& Sullivan (1978). Cette espèce parasite également $S$. avenae contenant Ephedrus et Praon (Rennes et Quimper, juillet 1970; Dadonville (Loiret) 24 juin 1974 et $M$. dirhodum contenant Praon (H le 14 juin 1976).

Deux espèces ont été trouvées assez fréquemment, mais en petits nombres: $D$. (M.) aphidum Rond. et $D$. (M.) rectangularis Kieff., qui ont été obtenues de momies récoltées chaque année pendant tout le mois de juin dans la moitié des parcelles étudiées. Ces espèces avaient été signalées sur S. avenae en Tchécoslovaquie (STARY, 1977).

D. (Atritomelius) laticeps Hedicke enfin n'a été trouvée qu'en une seule occasion (les 14 et 21 juin 1976). Elle avait été signalée sur $R$. padi (TAKADA, 1973).

\section{Cynipoidea - Cynipidae - Alloxystinae}

Les Alloxystinae sont endoparasites des Aphidiidés; l'œuf est pondu dans le parasite primaire, alors que le puceron est encore vivant.

Les 2 espèces largement dominantes, Alloxysta victrix Westw. et Phaenoglyphis villosa Htg., sont observées sur les 3 pucerons des céréales. Dans l'ensemble, leurs populations comportent 52 p. 100 de femelles pour $P$. villosa et 63 p. 100 pour $A$. victrix. A. victrix est considérée comme peu spécifique par EVENHUIS (1976) et par ANDREWS (1978), pouvant s'attaquer notamment aux Aphidius parasitant Macrosiphum et les genres voisins. $P$. villosa est également très polyphage et EVENHUIS \& BARBOTIN (1977) donnent une liste variée d'hôtes possibles.

Trois autres espèces n'ont été trouvées qu'exceptionnellement, sur $M$. dirhodum, en parcelles bocagères et à faible distance du bord des champs. Alloxysta castanea Kieff. est une espèce subaptère ; nous l'avons récoltée les 4 et 11 juin 1975. A. macrophadna Htg., hyperparasite fréquent d'Acyrthosiphon pisum Harr. (EVENHUIS, 1974) avait aussi été trouvé très exceptionnellement sur Cavariella aegopodii Scop. (RABASSE \& BRUNEL, 1977), il a été obtenu les 6 et 22 juin 1975. Enfin, Phaenoglyphis xanthochroa Forst. a été récolté le 17 juin de la même année; EvENHUIs (1978) pense que cette espèce pourrait être spécialisée dans l'hyperparasitisme de gros pucerons de type Macrosiphum.

\section{Chalcidoïdea - Pteromalidae}

Les Pteromalidés attaquent les Aphidiidés après formation de la momie ; ils sont ectoparasites des Aphiidés.

Asaphes vulgaris Wlk. est, de loin, le chalcidien le plus fréquent; nous l'avons trouvé dans presque toutes nos observations. Son sex-ratio est équilibré (pour l'ensemble des récoltes : 51 p. 100 de femelles). Cette espèce parasite également $M$. dirhodum contenant Praon $(\mathrm{H})$ et $R$. padi contenant Ephedrus.

Deux espèces sont souvent présentes sans que leurs effectifs soierit élevés. Asaphes suspensus Nees est habituellement très abondant dans les milieux cultivés et extrêmement polyphage (KAMIJO \& TAKADA, 1973), comme l'espèce précédente. Sur blé, il est moins fréquent qu' $A$. vulgaris et présente un sex-ratio équilibré $(53$ p. 100 de femelles). Nous avons obtenu Pachyneuron solitarium Htg. des 3 pucerons des céréales; cette espèce est présente pendant une longue période du 11 juin au 24 juillet en 1975, du 2 au 22 juin en 1976 et du 31 mai au 5 juillet en 1977. KamiJo \& TAKADA (1973) ont obtenu cette espèce de pucerons des arbres forestiers; ils la considèrent comme très polyphage, puisqu'elle est également hyperparasite de cochenilles et d'œufs de Lépidoptères. 
Enfin, 3 espèces sont relativement rares : Coruna clavata Wlk., qui a été trouvé assez abondamment par DEAN (1974) sur blé, n'a été obtenu qu'en 1975 dans des parcelles bocagères sur $M$. dirhodum et $S$. avenae entre le 11 et le 24 juin. Pachyneuron aphidis Bouché, également obtenu de ces 2 pucerons, n'a été trouvé que dans les environs de Rennes le 22 juin 1976 et le 22 juin 1977. Il s'agit d'une espèce très polyphage (KAMIJO \& TAKADA, 1973). Deux exemplaires de Pachyneuron concolor Forst. ont été trouvés en zones bocagères les 24 juin et 8 juillet 1975. Cette espèce, parasite de cochenilles, ne semble pas avoir été signalée sur pucerons jusqu'à présent (GRAHAM, 1969 ; BOUČEK, 1977).

\section{Importance relative des hyperparasites}

Il est difficile d'interpréter le pourcentage d'hyperparasitisme dans un échantillon de momies: Sullivan (1972) indique, en effet, qu'à $21^{\circ} \mathrm{C}$ le parasite primaire Aphidius smithi Sharma \& Subba Rao passe 4 j dans la momie, tandis que les hyperparasites y passent 2 à 3 fois plus longtemps. L'estimation de l'importance relative des hyperparasites présente également certaines difficultés. La ponte des hyperparasites ectophages se poursuit en effet pendant toute la présence de la momie et peut s'exercer aussi aux dépens des hyperparasites endophages. Le prélèvement de momies doit donc surestimer l'abondance finale des endoet sous-estimer celle des ectoparasites.

En tenant compte de cette remarque et du fait qu'ils sont présents dans toutes les parcelles, nous considérons que Dendrocerus carpenteri, Phaenoglyphis villosa, Alloxysta victrix et Asaphes vulgaris sont les principaux hyperparasites. Dendrocerus aphidum, Asaphes supensus et Pachyneuron solitarium jouent un rôle nettement moins important (tabl. 3).

\section{LES POPULATIONS DE PARASITES}

\section{A. Evolution dans le temps (fig. 1 et 2)}

Les parasites colonisent la culture très peu de temps après les pucerons. En 1975, dans les parcelles bocagères de Réguigny (C et D), le nombre de momies d'A. uzbekistanicus et d'A. picipes évolue peu du 15 mai au 15 juin. Dans la parcelle ouverte $\mathrm{E}$, située à proximité, les parasites semblent se manifester nettement plus tard. Dans les parcelles bocagères, $D$. carpenteri représente 10 p. 100 de l'effectif d'A. uzbekistanicus pendant un mois : du 15 mai au 15 juin. Dans les 3 champs, il élimine les parasites primaires fin juin. A Rennes (B), A. uzbekistanicus est moins abondant et disparaît plus tôt, tandis qu' $A$. picipes se maintient jusqu'à fin juillet; dans cette station, les températures moyennes suivent la même évolution qu'à Réquigny, tout en étant un peu plus élevées.

A Plonevez-Porzay (H), en 1976, du 15 mai au 15 juin, s'instaure un certain équilibre entre les différents hyperparasites et les parasites primaires $A$. uzbekistanicus et $A$. matricariae, dont les populations baissent graduellement.

En 1977, les températures sont basses et varient peu pendant la période d'observation ; les 4 exemples que nous possédons (I, J, K, L) présentent de grandes analogies et sont plus conformes à ce qui est observé habituellement sur d'autres cultures: le nombre de parasites croît jusqu'à la $3^{\mathrm{e}}$ semaine de juin, puis décroît. La courbe des hyperparasites (plusieurs espèces jouent un rôle important) suit avec un certain décalage.

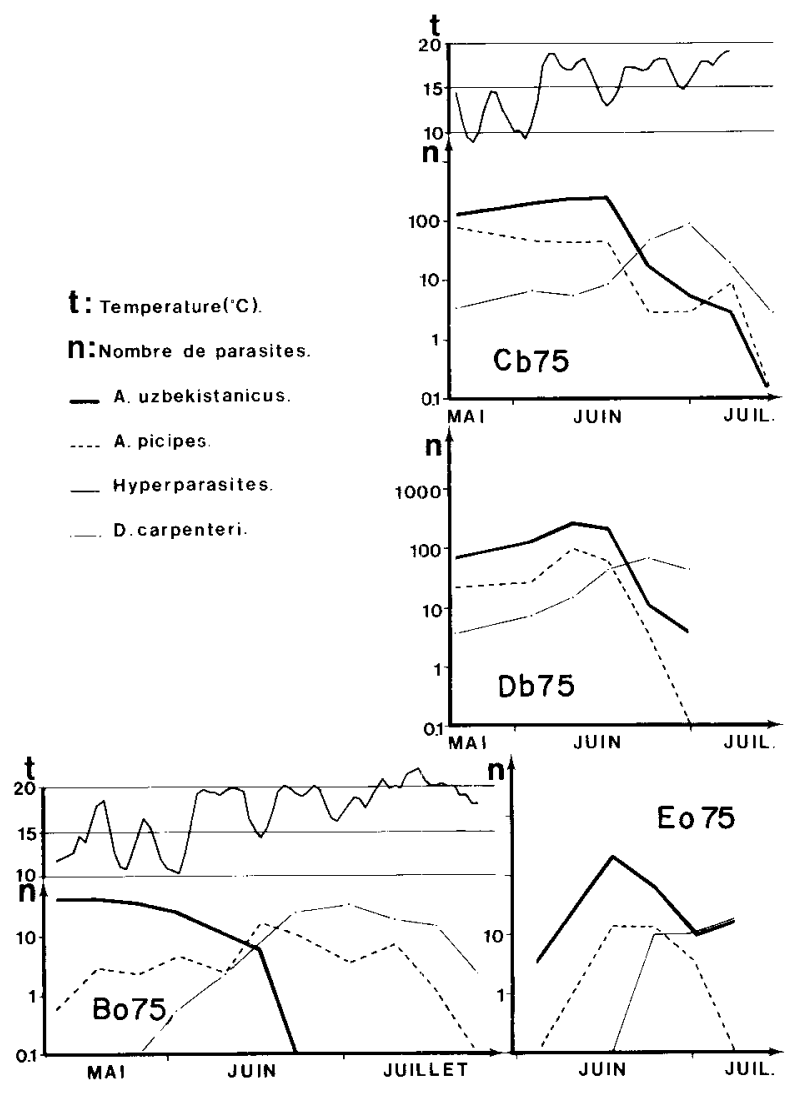

Figure 1

Evolution dans le temps du nombre de parasites présents dans les momies des différents aphides sur 1000 talles de blé en 1975. (Les parcelles sont désignées conformément au tableau 1.)

Les températures sont lissées sur une période de 3 jours. D. carpenteri est indiqué lorsque cet hyperparasite est largement dominant.

Number of parasites emerging from the mummies of different aphid species collected on 1000 tillers in 1975. (Plots are designated according to table 1.)

Temperatures averaged over 3-day periods. D. carpenteri is mentioned only when it is highly dominant.

Deux remarques peuvent être faites à l'observation de cet ensemble de courbes:

- les populations des 4 espèces d'Aphidius évoluent de la même façon dans une parcelle donnée, laissant penser qu'elles répondent aux mêmes facteurs essentiels (sauf à Rennes (B), en 1975, où $A$. picipes reste présent longtemps après $A$. uzbekistanicus);

- les populations d'hyperparasites augmentent brusquement en réponse à une élévation de température : en $1976(\mathrm{H})$, après une période au cours de laquelle la température moyenne quotidienne est aux alentours de $13{ }^{\circ} \mathrm{C}$ puis monte à $18-19^{\circ} \mathrm{C}$, les 7,8 et 9 juin, le prélèvement du 14 juin présente une forte augmentation des 3 hyperparasites $D$. carpenteri, $A$. vulgaris et A. victrix par rapport à celui du 8 juin.

\section{B. Localisation dans le champ}

Nous examinerons d'abord les différences d'activité des parasites au sein de la parcelle. Nous avons signalé plus haut que l'échantillonnage - commun pour les pucerons et leurs ennemis - avait été fait selon un système complexe comportant des prises de végétal identiques le long des transects dans des champs ouverts ou entourés de haies. En chaque point d'observation, l'information disponible est assez faible, si l'on veut tenir compte des différentes espèces 

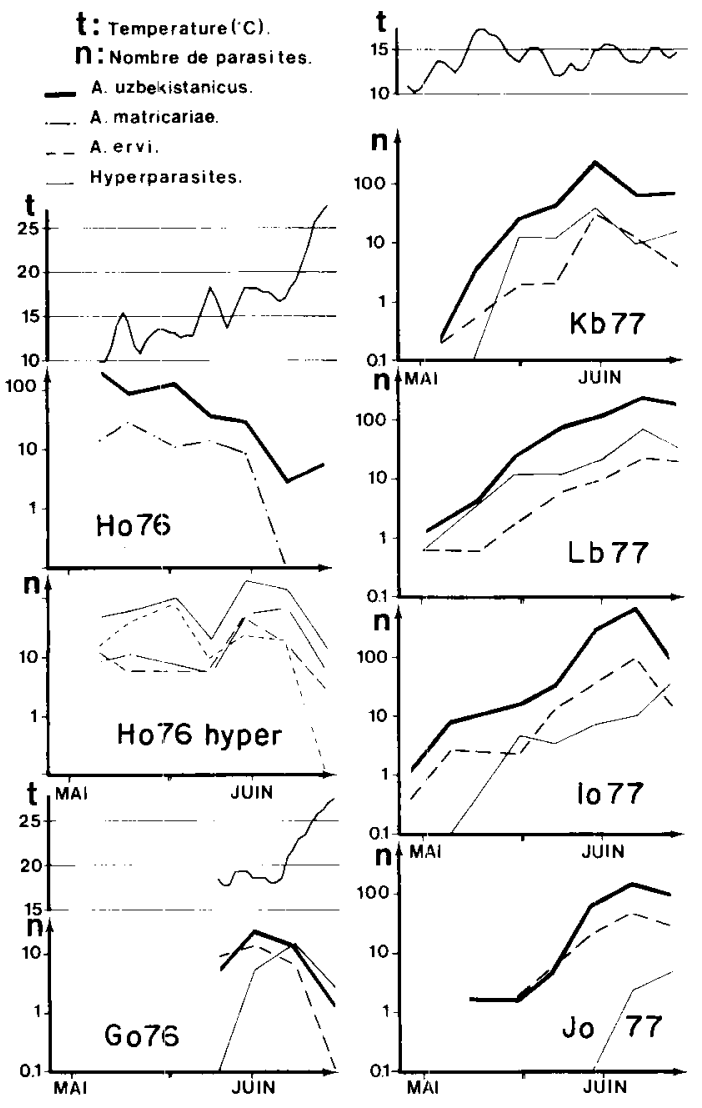

Figure 2

Evolution dans le temps du nombre de parasites présents dans les momies des différents aphides sur 1000 talles de blé en 1976 et 1977. Les températures sont lissées sur une période de 3 jours. Les hyperparasites ne sont détaillés que dans le graphique Ho 76 hyper (. - . D. carpenteri ; - - A. vulgaris; - - A. victrix).

Number of parasites emerging from the mummies of different aphid species collected on 1000 tillers in 1976 and 1977.

Temperatures averaged over 3-day periods. Hyperparasites shown in detail only in the graph Ho 76 hyper (. - . D. carpenteri ; - - A. vulgaris ; - - A. victrix).

de pucerons et de parasites, et les représentations graphiques des transects ou les tests d'homogénéité non paramétriques que nous avons tentés ne montrent pas de tendance bien nette. Le critère que nous avons pu retenir est le nombre d'Aphidius d'une espèce donnée obtenu d'échantillons de blé de même taille prélevés à différentes distances du bord de la parcelle. Le tableau 4 montre que, dans tous les champs ouverts, ce nombre diminue jusqu'à une centaine de mètres à l'intérieur de la parcelle. Dans les parcelles bocagères, c'est surtout une bande dont la largeur correspond à la moitié de la hauteur de la haie, soit environ $5 \mathrm{~m}$, qui produit nettement plus de parasites que le reste du champ.

Bien que ce résultat soit assez net, il faut reconnaitre qu'il n'explique pas grand chose. Quelle est la distance d'exploration efficace des parasites? Les taux de parasitisme sont-ils homogènes dans la parcelle? Autant de questions de grande importance, puisqu'elles conditionnent l'efficacité des parasites dans des parcelles de tailles différentes.

\section{Localisation sur la plante}

Les différents aphides ne sont pas répartis de façon uniforme sur les feuilles des plants de blé (DEDRYVER \& ROBERT, 1977); nous avons cherché à savoir si le parasi- tisme aussi se manifestait différemment selon les niveaux du végétal. Pour que la comparaison soit précise, nous avons dû la limiter à un puceron qui colonise bien tige et épi : $S$. avenae, aux dates où cette espèce est suffisamment abondante. Dans les parcelles choisies, en 1977, les momies doivent être attribuées en majorité à $A$. uzbekistanicus. Le tableau 5 montre que, en moyenne dans l'ensemble des cas étudiés, le pourcentage de momies dans les populations de $S$. avenae est 4 fois plus fort sur feuilles que sur épis. Deux raisons peuvent être invoquées pour expliquer les différences observées :

— la «montée sur épis » de $S$. avenae est un phénomène d'observation courante. Or, les momies, fixées à la plante, restent en place et sont les témoins d'un parasitisme passé, comme ROBERT (1979) a pu le montrer sur pomme de terre ;

- certains pucerons parasités ont tendance à s'isoler et à rechercher des sites particuliers. Par exemple, Acyrthosiphon pisum, parasité par Aphidius ervi Hal., $s$ 'isole à l'extrémité des folioles de légumineuses. $S$. avenae parasité présente peut être un comportement qui lui fait préférer les feuilles.

Cette observation a, bien entendu, des conséquences pour l'échantillonnage des populations de pucerons et de leurs parasites, mais elle ne traduit pas forcément une différence d'efficacité du parasite en fonction de la localisation $\mathrm{du}$ puceron.

\section{Spécificité}

Les données sur la spécificité des entomophages sont en général qualitatives et ce n'est qu'en conditions de laboratoire qu'il est possible d'apprécier la préférence pour tel ou tel hôte (JourdHEuIL, 1978). Etant en présence de 3 parasites primaires (A. uzbekistanicus, A. ervi, A. picipes) capables d'attaquer 2 pucerons ( $M$. dirhodum et $S$. avenae), nous avons cherché à mettre en évidence une préférence de tel parasite pour tel puceron, le critère d'appréciation étant le nombre de parasites effectivement éclos d'une espèce de puceron, c'est-à-dire le résultat final au champ de l'action de l'entomophage. Si nous disposions de forts effectifs de pucerons et de momies de chaque catégorie, il suffirait de comparer les pourcentages de momies formées par chaque parasite. Ce n'est évidemment pas le cas; aussi notre objectif se limite-t-il à mettre une préférence en évidence lorsque c'est statistiquement possible. Dans ce but, nous avons établi des tableaux croisés comportant les effectifs de $M$. dirhodum et de $S$. avenae sains ou parasités par une espèce donnée à une date donnée. Nous avons alors calculé les indices d'association de BORDET \& ALBUISSON (1974) et leur signification. Cette méthode permet le traitement de données brutes sans contrainte de normalité des populations. Parmi 26 tableaux concernant A. uzbekistanicus, 14 concernant $A$. ervi et $8 A$. picipes, nous n'avons retenu que les résultats significatifs (tabl. 6). Les indices d'association nuls, qui traduisent simplement un faible parasitisme, n'ont pas été indiqués. Dans 3 cas, A. picipes présente une forte association positive avec $S$. avenae. Il n'a pas été possible de mettre en évidence d'association d'A. ervi avec l'un ou l'autre aphide. Enfin, en 1975, A. uzbekistanicus présente une association positive avec $S$. avenae et négative avec $M$. dirhodum en début de saison, puis, progressivement, cette tendance s'inverse. S'il est logique de mettre ce changement en relation avec la « montée sur épis» de $S$. avenae, il serait sans doute hasardeux de vouloir l'interpréter trop étroitement. En effet, pour des raisons pratiques, nous avons mis en relation les effectifs de momics et de pucerons obscrvés à 
TABLEAU 4

Nombre d'Aphidius éclos des momies de pucerons récoltées sur des échantillons de blé de même taille prélevés à différentes distances du bord de la parcelle.

Numbers of Aphidius emerged from mummies collected in samples containing the same number of tillers and cut at different distances from the edge of the field.

Aphidius uzbekistanicus Luz.

\begin{tabular}{|c|c|c|c|c|c|c|c|}
\hline \multirow[t]{2}{*}{ Parcelle } & \multirow[t]{2}{*}{ Période } & \multicolumn{6}{|c|}{ Distance } \\
\hline & & $1 / 2 \mathrm{~h}\left({ }^{*}\right)$ & $1 \mathrm{~h}$ & $2 \mathrm{~h}$ & $4 \mathrm{~h}$ & $6 \mathrm{~h}$ & \\
\hline $\mathrm{Cb} 75$ & $22 / 5-24 / 6$ & 91 & 39 & 52 & 48 & 34 & \\
\hline \multirow[t]{2}{*}{ D b 75} & $22 / 5-24 / 6$ & 46 & 36 & 31 & 34 & 30 & \\
\hline & & Bord & $(10-40 \mathrm{~m})$ & $(50-80 \mathrm{~m})$ & $(90-120 \mathrm{~m})$ & $(130-160 \mathrm{~m})$ & $(170-200 \mathrm{~m})$ \\
\hline H o 76 & $20 / 5-25 / 5$ & $(54)$ & 32 & 26 & - & - & - \\
\hline I o 77 & $13 / 5-27 / 6$ & $(224)$ & 160 & 106 & 64 & 52 & 83 \\
\hline J o 77 & $13 / 6-27 / 6$ & (112) & 87 & 52 & 54 & 29 & 31 \\
\hline $\mathrm{K}$ b 77 & $1 / 6-28 / 6$ & - & 89 & 77 & 44 & - & - \\
\hline
\end{tabular}

Aphidius ervi Hal.

\begin{tabular}{lccccccc}
\hline \hline & Bord & $(10-40 \mathrm{~m})$ & $(50-80 \mathrm{~m})$ & $(90-120 \mathrm{~m})$ & $(130-160 \mathrm{~m})$ & $(170-200 \mathrm{~m})$ \\
\hline I o 77 & $13 / 5-27 / 6$ & $(52)$ & 28 & 11 & 7 & 11 & 5 \\
J o 77 & $13 / 6-27 / 6$ & $(32)$ & 30 & 16 & 11 & 5 \\
\hline
\end{tabular}

(*) Pour les parcelles bocagères $\mathrm{C}$ et $\mathrm{D}$, les distances sont mesurées en multiples de la hauteur de la haie qui borde la parcelle (h).

- En général un prélèvement a été effectué tous les $10 \mathrm{~m}$; la valeur indiquée entre parenthèses pour le bord de la parcelle a donc été multipliée par un coefficient approprié (de 2 à 4 ) pour ramener l'échantillon à un même volume de végétal que pour les autres distances.

TABLEAU 5

Localisation des momies de Sitobion avenae $F$. sur les plants de blé.

Localization of the mummies of Sitobion avenae $F$. on the wheat plants.

\begin{tabular}{|c|c|c|c|c|c|c|}
\hline \multirow{2}{*}{ Parcelle } & \multirow{2}{*}{ Date } & \multirow{2}{*}{$\begin{array}{c}\text { Stade } \\
\text { (échelle de FeEKEs) }\end{array}$} & \multirow{2}{*}{$\begin{array}{c}\text { Nombre de talles } \\
\text { observées }\end{array}$} & \multirow{2}{*}{$\begin{array}{l}\text { Nombre d'aphides } \\
\text { sains observés }\end{array}$} & \multicolumn{2}{|c|}{$\%$ de pucerons momifiés } \\
\hline & & & & & Sur feuilles & Sur épis \\
\hline I o 77 & $\begin{array}{r}7-6 \\
14-6 \\
21-6 \\
28-6\end{array}$ & $\begin{array}{l}10,5 \\
10,5,4 \\
11,10 \\
11,20\end{array}$ & $\begin{array}{l}840 \\
840 \\
200 \\
200\end{array}$ & $\begin{array}{r}14443 \\
26110 \\
8514 \\
1529\end{array}$ & $\begin{array}{l}0,42 \\
2,9 \\
7,9 \\
8,6\end{array}$ & $\begin{array}{l}0,26 \\
0,82 \\
1,9 \\
2,4\end{array}$ \\
\hline J o 77 & $\begin{array}{l}13-6 \\
20-6 \\
27-6\end{array}$ & $\begin{array}{l}10,5.4 \\
11,10 \\
11,20\end{array}$ & $\begin{array}{l}840 \\
840 \\
840\end{array}$ & $\begin{array}{r}10981 \\
14597 \\
6928\end{array}$ & $\begin{array}{l}2,0 \\
5,5 \\
4,1\end{array}$ & $\begin{array}{l}0,40 \\
1,1 \\
2,5\end{array}$ \\
\hline K b 77 & $\begin{array}{r}7-6 \\
14-6 \\
21-6 \\
28-6\end{array}$ & $\begin{array}{l}10,5 \\
10,5.4 \\
11,10 \\
11,20\end{array}$ & $\begin{array}{l}520 \\
520 \\
520 \\
520\end{array}$ & $\begin{array}{r}6072 \\
16501 \\
20441 \\
4832\end{array}$ & $\begin{array}{r}1,4 \\
3,3 \\
2,8 \\
11,4\end{array}$ & $\begin{array}{l}0,21 \\
0,96 \\
0,71 \\
2,3\end{array}$ \\
\hline
\end{tabular}

la même date, alors qu'il faudrait utiliser le nombre de pucerons présents au moment où a eu lieu le parasitisme (une semaine avant?) pour ne pas être trop dépendants des fluctuations de populations. De plus, nous n'avons pas tenu compte de la couleur (vert ou brun) de $S$. avenae, qui semble jouer un rôle lors du choix d'A. uzbekistanicus (ANKERSMIT et al., 1981).

Remarquons enfin que seulement 3 espèces d'aphides hôtes ont été mentionnées dans cette étude. Il est cependant possible que quelques Metopolophium festucae Theob. soient regroupés sous notre dénomination $M$. dirhodum et quelques Sitobion fragariae Wlk. sous le nom $S$. avenae. En effet, à notre connaissance, il n'est pas possible actuellement de reconnaître ces espèces sur des momies présentant la forme larvaire du puceron. Seulement 4 momies ont pu être attribuées à l'espèce $S$. fragariae: elles étaient parasi- 


\section{TABLEAU 6}

Indice d'association de BORDET \& ALBUISSON entre l'effectif des pucerons sains et le nombre de momies dans l'échantillon. L'indice de signification est mentionné entre parenthèses.

BORDET dE ALBUISSON association index between the number of healthy aphids and the number of mummies in the sample.

\begin{tabular}{|c|c|c|c|c|}
\hline & Parcelle & Date & M. dirhodum & S. avenae \\
\hline \multirow[t]{8}{*}{ A. uzbekistanicus } & & & & ++++ \\
\hline & B o 75 & $20-5$ & --- & $(++++)$ \\
\hline & $\mathrm{C} \mathrm{b} 75$ & $22-5$ & & $\begin{array}{c}++ \\
(+++)\end{array}$ \\
\hline & & $17-6$ & & $\stackrel{-}{(-)}$ \\
\hline & D b 75 & $17-6$ & & $\begin{array}{c}-- \\
(----)\end{array}$ \\
\hline & F o 75 & $17-6$ & $\begin{array}{c}++ \\
(++++)\end{array}$ & \\
\hline & E o 75 & $17-6$ & $\begin{array}{c}+++ \\
(+++)\end{array}$ & $\begin{array}{c}--- \\
(----)\end{array}$ \\
\hline & & $24-6$ & $\begin{array}{c}++ \\
(++++)\end{array}$ & \\
\hline
\end{tabular}

A. picipes

$\begin{array}{ccc}\text { C b } 75 & 22-5 & ++ \\ & 4-6 & (++) \\ & & ++ \\ \text { D b } 75 & 4-6 & (+++) \\ & +++ \\ & & (+++)\end{array}$

Indice d'association : - - - : $-0,75 ;---:-0,50 ;--:-0,20 ;-:-0,10 ;+0,10:+;+0,20:++;+0,50:+++$;

$+0,75:+++$.

Indice de signification: $----: 0,0010 ;---: 0,0025 ;--: 0,0050 ;-: 0,0100 ; 0,9900:+; 0,9950:++; 0,9975:+++$; $0,9990:+++$.

TABLEAU 7

Nombre de mâles et de femelles d'Aphidius uzbekistanicus Luz. et tests de conformité aux proportions théoriques de 50 p. 100 et 40 p. 100 de mâles dans la population.

Numbers of Aphidius uzbekistanicus Luz. males and females and $\chi^{2}$-tests for conformity to the theoretical ratios $50 \%$ and $40 \%$ males.

\begin{tabular}{|c|c|c|c|c|c|c|}
\hline Parasites & Parcelle & $\delta$ & q & $\begin{array}{c}\chi^{2} 50 \% \\
\mathrm{dc} \delta\end{array}$ & $\begin{array}{c}x^{2} 40 \% \\
\operatorname{de} \delta\end{array}$ & Hôtes \\
\hline A. uzbekistanicus & $\begin{array}{l}\text { B o } 75 \\
\text { C b } 75 \\
\text { D b } 75 \\
\text { E o } 75 \\
\text { H o } 76 \\
\text { B o } 75 \\
\text { C b } 75 \\
\text { I o } 77 \\
\text { J o } 77 \\
\text { K b } 77 \\
\text { L b } 77\end{array}$ & $\begin{array}{r}54 \\
95 \\
58 \\
12 \\
22 \\
24 \\
13 \\
184 \\
93 \\
87 \\
157\end{array}$ & $\begin{array}{r}110 \\
139 \\
106 \\
38 \\
38 \\
69 \\
33 \\
317 \\
166 \\
131 \\
234\end{array}$ & $\begin{array}{r}19,1 \\
8,3 \\
14,0 \\
13,5 \\
4,3 \\
21,8 \\
8,7 \\
35,3 \\
20,6 \\
8,9 \\
15,2\end{array}$ & $\begin{array}{l}3,4 \\
0,03 \\
1,5 \\
5,3 \\
0,3 \\
7,8 \\
2,6 \\
2,2 \\
1,8 \\
<0,1 \\
<0,1\end{array}$ & $\begin{array}{l}\text { M. dirhodum } \\
\text { M. dirhodum } \\
\text { M. dirhodum } \\
\text { M. dirhodum } \\
\text { M. dirhodum } \\
\text { S. avenac } \\
\text { S. avenac } \\
\text { S. avenac } \\
\text { S. avenac } \\
\text { S. avenac } \\
\text { S. avenac }\end{array}$ \\
\hline A. ervi & $\begin{array}{l}\text { I o } 77 \\
\text { J o } 77 \\
\text { L b } 77\end{array}$ & $\begin{array}{l}34 \\
26 \\
10\end{array}$ & $\begin{array}{l}42 \\
53 \\
23\end{array}$ & $\begin{array}{l}0,8 \\
9,2 \\
5,1\end{array}$ & $\begin{array}{l}0,7 \\
1,7 \\
1,3\end{array}$ & $\begin{array}{l}\text { S. avenac } \\
\text { S. avenac } \\
\text { S. avenac }\end{array}$ \\
\hline A. picipes & $\begin{array}{l}\text { C b } 75 \\
\text { D b 75 } \\
\text { B o } 75\end{array}$ & $\begin{array}{l}19 \\
24 \\
17\end{array}$ & $\begin{array}{l}26 \\
23 \\
18\end{array}$ & $\begin{array}{l}1,1 \\
0,1 \\
0,02\end{array}$ & $\begin{array}{l}0,1 \\
2,4 \\
1,1\end{array}$ & $\begin{array}{l}\text { M. dirhodum } \\
\text { M. dirhodum } \\
\text { S. avenac }\end{array}$ \\
\hline A. matricariae & Ho 76 & 25 & 43 & 4,8 & 0,3 & R. padi \\
\hline
\end{tabular}

$\chi^{2} 0,05=3,84$. 
tées par A. uzbekistanicus (6 mai 1975, B et 11 juin 1975, C) et par $A$. picipes (11 juin $1975, C$ ). D'après ce que nous savons de la spécificité des Aphidiidés, les parasites sont généralement les mêmes pour les espèces d'un même genre ayant un mode de vie voisin sur la même plante-hôte.

\section{E. Sex-ratio}

Plusieurs auteurs ont constaté le déséquilibre du sex-ratio des Aphidiidés (MACKAUER, 1976a ; RABASSE \& BRUNEL, $1977)$ et ont essayé de lui trouver des explications théoriques (MACKAUER, 1976b).

Nous disposons d'un ensemble de données important sur les principaux parasites des pucerons des céréales. Le sexratio est ici apprécié sur les éclosions effectives de momies prélevées au champ. Nous ne reproduisons pas les courbes d'évolution dans le temps de ce rapport, tracées pour $A$. uzbekistanicus dans les essais $\mathrm{B}$, I et $\mathrm{L}$ : elles ne montrent pas de tendance évidente. Nous avons donc regroupé l'ensemble des éclosions obtenues dans une parcelle au cours d'une saison sur un puceron-hôte donné.
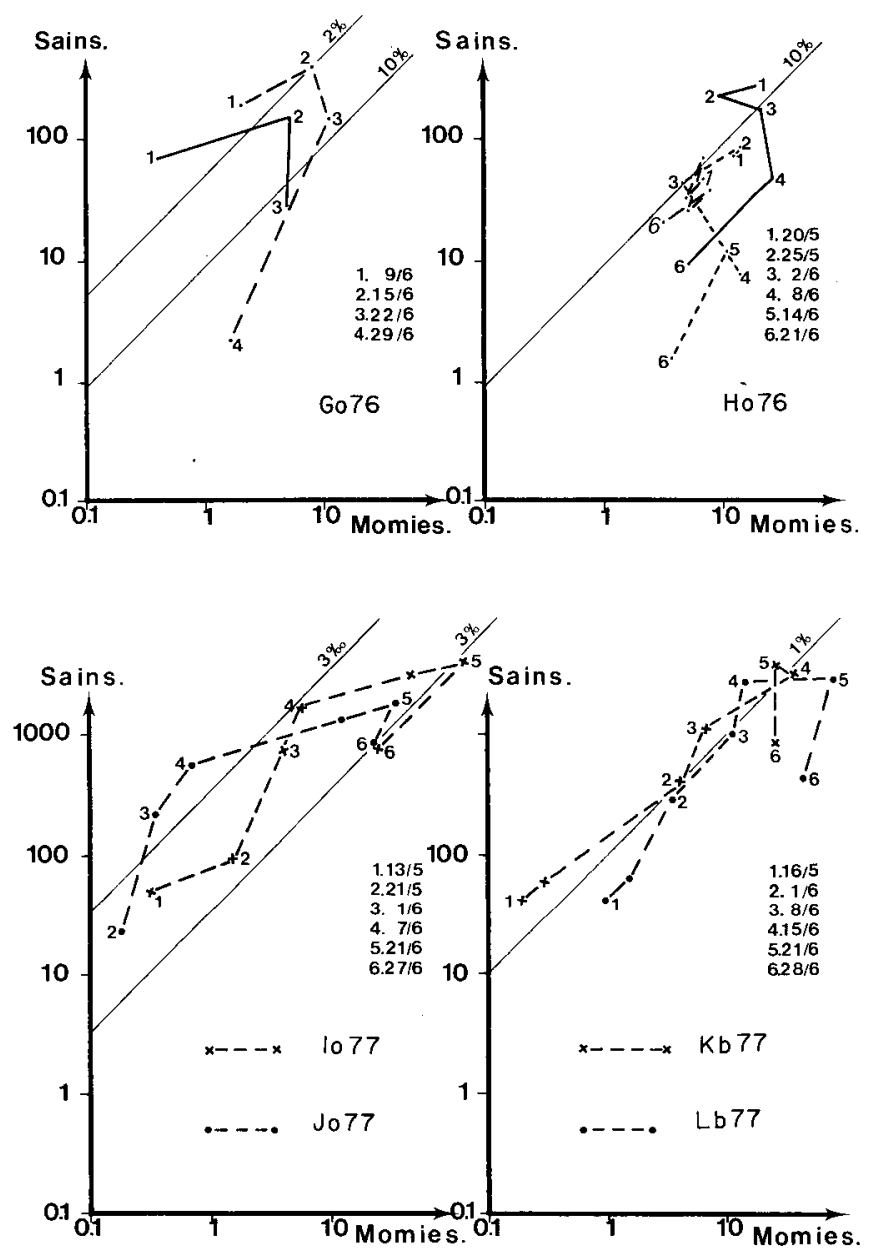

---S. avenae - M.dirhodum .......R. padi

Figure 3

Evolution dans le temps de la relation entre le nombre de pucerons sains et le nombre de momies en 1975 (valeurs pour 100 talles). Les parcelles sont désignées conformément au tableau 1. Les observations sont hebdomadaires.

Development in time of the relationship between the number of living aphids and the number of mummies per 100 tillers in 1975 (Values per 100 tillers).

Plots are designated according to table 1. Weekly observations.
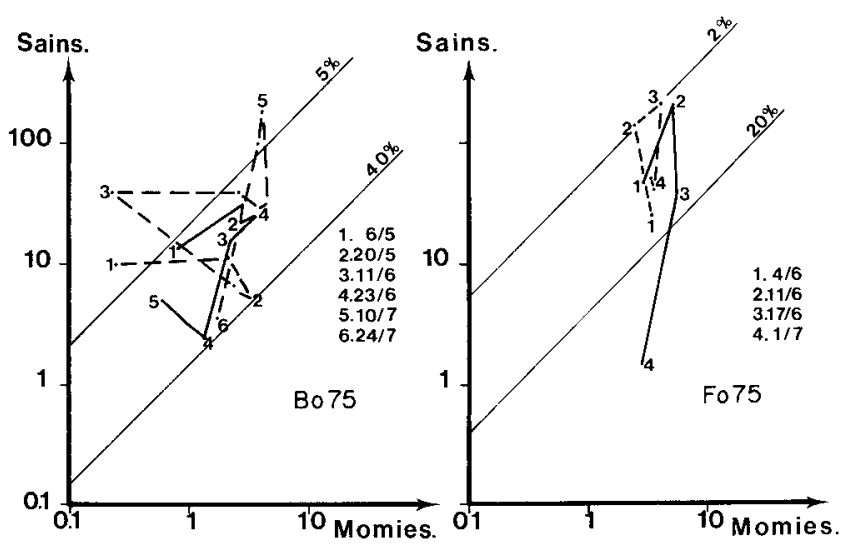

Pour l'ensemble des parasites éclos, nous obtenons les pourcentages suivants de femelles:

A. uzbekistanicus sur $R$. padi: 61 p. 100.

A. uzbekistanicus sur $M$. dirhodum: 66 p. 100.

A. uzbekistanicus sur S. avenae: 63 p. 100.

A. ervi sur $S$. avenae : 60 p. 100.

A. picipes sur $S$. avenae et $M$. dirhodum: 52 p. 100.

A. matricariae sur $R$. padi: 63 p. 100.

Ces résultats d'ensemble nous ont donc conduits à tester dans les différentes conditions où nous avions obtenu plus de 30 parasites les 2 hypothèses : 50 p. 100 et 40 p. 100 de mâles (tabl. 7). Pour A. uzbekistanicus, l'hypothèse d'un sex-ratio équilibré est toujours contredite, tandis que l'hypothèse 40 p. 100 de mâles n'est refusée que dans 2 cas : en 1975, à Moreac (E) sur $M$. dirhodum et à Rennes (B) sur $A$. avenae. Pour $A$. ervi, l'hypothèse 40 p. 100 n'est jamais repoussée, tandis que l'hypothèse 50 p. 100 est refusée dans 2 cas sur 3 . Dans le cas d'A. picipes, aucune hypothèse n'est repoussée, mais les effectifs sont assez faibles. Enfin, $S$. matricariae présente 40 p. 100 de mâles, ce qui confirme les observations faites sur d'autres pucerons-hôtes (SHALABY \& RABASSE, 1979).

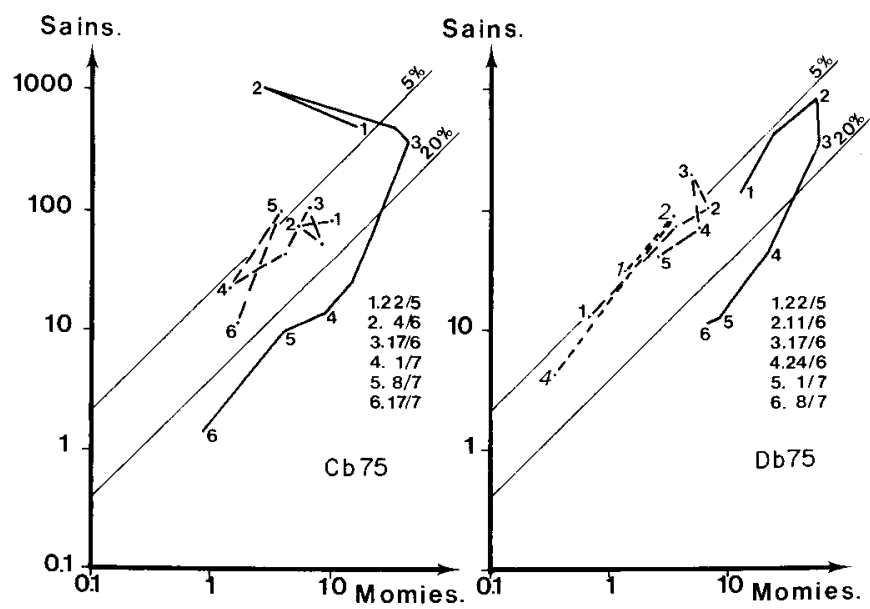

Figure 4

Evolution dans le temps de la relation entre le nombre de pucerons sains et le nombre de momies en 1976 et 1977 (valeurs pour 100 talles).

Les observations sont hebdomadaires.

Development in time of the relationship between the number of living aphids and the number of mummies per 100 tillers in 1976 and 1977 (Values per 100 tillers).

Weekly observations. 
Ces observations établissent donc le déséquilibre du sexratio à l'éclosion des momies pour $A$. uzbekistanicus, dans des conditions variées. Ce même déséquilibre est confirmé pour $A$. ervi et $A$. matricariae, tandis que le cas d' $A$. picipes pourrait fort bien être différent.

\section{LIMITATION DES POPULATIONS DE PUCERONS}

Les figures 3 et 4 présentent l'évolution dans le temps de la relation entre les populations de pucerons et le parasitisme exprimé par le nombre de momies, qui traduit essentiellement l'action d'A. uzbekistanicus (comme le montrent les figures 1 et 2 ). On peut distinguer plusieurs cas :

- En 1975. dans les champs ouverts (B, F), les populations aphidiennes n'atteignent pas un niveau élevé. Elles sont limitées à partir du début mai à Rennes (B) par l'action des parasites et accessoirement des Entomophthorales ; fin mai-début juin, on observe 12 p. 100 de momies sur $M$. dirhodum et 30 p. 100 sur $S$. avenae. En juin, cependant, le nombre de momies stagne et le parasitisme ne semble pas capable d'enrayer une lente remontée des populations de $S$. avenae; la figure 1 permet d'attribuer la limitation de l'action d'A. uzbekistanicus à cette époque aux hyperparasites qui profitent des températures élevées. A Moreac (F), la population de pucerons semble évoluer indépendamment du nombre de parasites, qui reste assez constant ; le 17 juin, on note au maximum 13 p. 100 de momies sur $M$. dirhodum, alors que les populations de pucerons sont en pleine régression.

- Dans les parcelles bocagères, par contre, à Reguigny (C et $\mathrm{D})$, la population de $M$. dirhodum, qui est à un niveau élevé lors des premières observations, diminue fortement, limitée par les parasites et les mycoses dans la $1^{\text {re }}$ quinzaine de juin: le 17 juin, on observe 9 p. 100 de momies et 19 p. 100 de mycoses dans la parcelle $C$ et 12 p. 100 de momies et 8 p. 100 de mycoses dans la parcelle $D$. Les courbes relatives à $S$. avenae sont soit très irrégulières $(\mathrm{C})$, soit caractéristiques d'un taux de parasitisme constant : dans la parcelle $\mathrm{D}$, on observe $5 \mathrm{p} .100$ de momies, tant pour $S$. avenae que pour $R$. padi. Il sernble donc que $A$. uzbekistanicus, même produit en abondance sur $M$. dirhodum, ne réussisse pas à limiter franchement $S$. avenae.

- En 1976, les exemples de Rennes (G) et de PlonevezPorzay $(\mathbf{H})$ sont extrêmement différents. A Rennes, il n'y a pas de mycose du fait de la sécheresse et de la chaleur exceptionnelle de 1976 (fig. 2). La croissance des populations de pucerons est limitée après le 15 juin, alors que le taux de momies - inférieur à 3 p. 100 - ne peut être invoqué pour expliquer ce phénomène. A PlonevezPorzay $(\mathrm{H})$, par contre, en raison de l'humidité maritime, une épizootie à Erynia neoaphidis Remaudière \& Henn. (DEDRYVER, 1978b) élimine les aphides; le nombre de pucerons sains des 3 espèces régresse, tandis que le nombre de momies reste constant, de l'ordre de 10 momies pour 100 talles.

- En 1977, S. avenae est de loin le puceron le plus abondant. A Rennes (I et J), on observe du 6 au 21 juin une augmentation progressive du taux de parasitisme, qui correspond à un abaissement des températures au-dessous de $15^{\circ} \mathrm{C}$ (fig. 2). Cependant, comme l'année précédente dans la' même parcelle, le parasitisme atteint un niveau bien trop faible pour constituer un facteur limitant décisif (au maximum, 3,5 p. 100 de momies le 21 juin dans la parcelle I). Dans les parcelles bocagères $\mathrm{K}$ et $\mathrm{L}$, le pourcentage de momies reste constant, de l'ordre de 1 p. 100 ; il n'augmente que dans les derniers jours de juin, mais du fait de la chute de la population saine, non d'une multiplication des parasites. En 1977, les mycoses ont contribué pour une large part à la limitation des populations.

\section{DISCUSSION, CONCLUSION}

Les espèces de parasites primaires que nous avons observées avaient déjà été signalées dans notre pays sur pucerons des graminées (STARÝ et al., 1973). L'image de leur importance relative que nous avons donnée pour le blé d'hiver en Bretagne est très voisine de celle obtenue par LATTEUR (1973) en Belgique, par DEAN (1974) \& CARTER et al. (1980) en Grande-Bretagne et par STARÝ (1976) en Tchécoslovaquie.

Parmi les hyperparasites, DEAN (1974) \& CARTER et al. (1980) avaient également trouvé que $D$. carpenteri était l'espèce la plus abondante, tandis que LATTEUR (1973) signalait le même complexe d'espèces de Dendrocerus. Si A. uzbekistanicus semble avoir des liens assez étroits avec les graminées, il n'en est pas de même des hyperparasites. Nous comparerons donc le cortège des hyperparasites des pucerons du blé à celui de $C$. aegopodii sur carotte, qui est notre principale référence en Bretagne (RABASSE \& BRUNEL, 1977) et d'Aphis fabae Scop. (RABASSE, non publié) sur féverole. Sur cette dernière culture, $D$. carpenteri est le mégaspilidé dominant, comme sur le blé, alors que $D$. laticeps est l'espèce la plus abondante sur carotte. Les Alloxystinae des pucerons des céréales sont nettement différents de ceux que nous avions trouvés sur artichaut (ROBERT et al., 1972) et carotte, où Alloxysta tscheki Giraud était l'espèce largement dominante. Ceci n'est d'ailleurs pas étonnant dans la mesure où la spécificité de ces parasites dépend à la fois de l'espèce de puceron et de l'espèce de parasite primaire (GUTIERREZ, $1970 a$ et $b$ ). Sur blé, Asaphes suspensus est moins fréquent qu'A. vulgaris, alors que ces 2 espèces présentaient des effectifs voisins sur carotte et fève. Enfin, Pachyneuron aphidis, qui était le ptéromalidé le plus abondant sur $A$. fabae et que nous avions trouvé en grand nombre sur $C$. aegopodii, est ici peu représenté, probablement parce que, sur blé, les pucerons parasités ne sont pas présents assez tard.

L'observation des pourcentages de memies montre que les aphidiidés, et tout particulièrement $A$. uzbekistanicus, s'implantent régulièrement dans les champs de céréales de l'ouest de la France et agissent sur les populations de pucerons. Cette dernière espèce peut d'ailleurs passer régulièrement l'hiver sur repousses de blé (RABASSE \& DEDRYVER, 1982). L'abondance relative des autres Aphidius varie selon les années, plutôt que selon les lieux. Les parasites apparaissent dans le champ en même temps que les pucerons. Malgré cela, leur action n'est pas déterminante : on observe en général nettement moins de 10 p. 100 de momies lors du pic de population, ce qui est cohérent avec les résultats obtenus en Hollande (ANKERSMIT \& CARTER, 1981) ; dans quelques cas, on atteint 20 p. 100. Mais surtout, les parasites ne semblent pas capables de se multiplier plus vite que leurs hôtes et donc, puisqu'il s'agit de parasites solitaires, sont incapables de les limiter efficacement. Cette analyse très schématique est, dans l'ensemble, applicable aux données obtenues par LATTEUR (1973) et LATTEUR \& DESTAIN (1980) en Belgique, par BODE (1980) en Allemagne et par DEAN (1974) en GrandeBretagne au cours de la dernière décennie. 
A. uzbekistanicus est donc le seul parasite relativement abondant et il n'a pas une efficacité aussi manifeste que d'autres parasites de pucerons sur d'autres cultures. Malgré notre manque de connaissances sur la bio-écologie de ces espèces, la comparaison des populations d'A. uzbekistanicus à celles d'A. salicis sur le puceron de la carotte ne manque pas d'intérêt: tandis que la densité de momies atteint $2000 / \mathrm{m}^{2}$ sur carotte, elle ne dépasse pas, même dans les cas de forte infestation en pucerons, $120 / \mathrm{m}^{2}$ sur céréales. $\mathrm{Ce}$ dernier chiffre doit être rapproché du nombre d'Aphidius spp. obtenus par JONES \& DEAN (1975) dans un piège à émergence sur blé : pendant plusieurs années, ces auteurs ont capturé de 28 à 102 individus $/ \mathrm{m}^{2}$ pendant toute la saison. Les Aphidius ont des fécondités élevées en laboratoire où ils peuvent former plusieurs centaines de momies par femelle. Leur descendance sur le terrain dépend étroitement de leurs conditions de vie (alimentation notamment) et du temps qu'ils consacrent à la recherche de leur hôte. Il est donc probable que la densité de l'hôte est un élément important de l'efficacité de ces entomophages. Sur carotte, au pic de population, les densités de 25000 pucerons $/ \mathrm{m}^{2}$ sont courantes. Sur blé, elles sont exceptionnelles (RABBINGE et al., 1979) et, en Bretagne, la densité maximale en année normale est 5 fois moins élevée. De plus, le volume de végétal à explorer est beaucoup plus grand sur céréales que sur carotte pour une même surface au sol.

L'action d'A. uzbekistanicus est quantifiée de façon très imprécise. Provisoirement, on introduit dans les modèles de populations aphidiennes sur céréales une mortalité due au parasitisme égale au double de l'effectif de momies (CARTER \& DiXon, 1981). On peut cependant comparer son mode d'action à celui d'autres parasites de pucerons. Sur carotte en plein champ ou sur aubergine en culture en serre (RABASSE et al., 1982), d'autres Aphidius présentent un effectif qui augmente rapidement et régulièrement. Par contre, sur céréales, A. uzbekistanicus se multiplie lentement et de façon irrégulière. Or, la période de présence des pucerons sur céréales est courte par rapport à la durée du cycle du parasite, qui est de l'ordre de $24 \mathrm{j}$ à $15^{\circ} \mathrm{C}$ (DRANSFIELD, 1979). On peut donc interpréter nos observations en disant qu'A. uzbekistanicus se comporte comme un agent de régulation à action discontinue et que son abondance ou son activité présente de fortes fluctuations à l'intérieur de la période où il peut avoir une action efficace.

Reçu le 7 janvier 1983 Accepté le 14 avril 1983.

\section{REMERCIEMENTS}

Les auteurs remercient vivement pour leurs identifications P. Dessart (Bruxelles, Megaspilidae), F. Barbotin (St Malo, Cynipidae), Z. BOUĆEK (Londres, Pachyneuron concolor), ainsi que P. Starý (Prague) et G. LatTeur (Gembloux) pour des précisions sur $A$. rhopalosiphi, enfin L. LAPCHIN (Antibes) pour ses indications concernant l'indice de BORDET.

\section{RÉFÉRENCES BIBLIOGRAPHIQUES}

Andrews F. G., 1978. Taxonomy and host specificity of nearctic Alloxystinae with a catalog of the world species (Hymenoptera, Cynipidae). California Depart. Agric. Occ. papers in Entomol., 25, $128 \mathrm{p}$.

Ankersmit G. W., Acreman T. M., Dijkman H., 1981. Parasitism of colour forms in Sitobion avenae. Entomol. exp. appl., 29, 362-363. Ankersmit G. W., Carter N., 1981. Comparison of the epidemiology of Metopolophium dirhodum and Sitobion avenae on winter wheat. Neth. J. Plant Pathol, 87, 71-81.

Bennett A. W., Sullivan D. J., 1978. Defensive behavior against tertiary parasitism by the larvae of Dendrocerus carpenteri an aphid hyperparasitoid. J. N.Y. Entomol. Soc., 86, 153-160.

Bode E., 1980. Aphids in winter wheat : abundance and limiting factors from 1976 to 1979. Bull. O.I.L.B./S.R.O.P., III/4, 49-57. Bordet J.-P., Albuisson M., 1974. Méthodes de dépouillement de tableaux croisés. Note interne ARLAB, 25, $56 \mathrm{p}$.

Boucek Z., 1977. A faunistic review of the Yugoslavian Chalcidoidea (parasitic Hymenoptera). Acta entomol. Jugosl., 13 suppl., 47. Carter N., Dixon A. F. G., 1981. The " natural enemy ravine" in cereal aphid population dynamics: a consequence of predator activity or aphid biology? J. anim. Ecol, 50, 605-611.

Carter N., McLean I. F. G., Watt A. D., Dixon A. F. G., 1980. Cereal aphids : a case study and review. Appl. Biol., 5, 271-348. Dean G. J., 1974. Effects of parasites and predators on the cereal aphids Metopolophium dirhodum (Wlk.) and Macrosiphum avenae (F.) (Hem., Aphididae). Bull. entomol. Res., 63, 411-422.

Dedryver C.-A., 1978a. Biologie des pucerons des céréales dans l'Ouest de la France. I. Répartition et évolution des populations de Sitobion avenae F., Metopolophium dirhodum Wlk. et Rhopalosiphum padi L., de 1974 à 1977 sur blé d'hiver dans le Bassin de Rennes. Ann. Zool. Ecol. anim., 10, 483-505.

Dedryver C.-A., 1978b. Rôle de l'humidité relative sur le développement du champignon parasite, Entomophthora aphidis Hoffmann, dans les populations de l'aphide, Sitobion avenae F., en 1976, dans l'Ouest de la France. C.R. Acad. Sc. Paris, sér. D, 286, 1723-1726.
Dedryver C.-A., 1981. Biologie des pucerons des céréales dans l'Ouest de la France. II. Répartition spatio-temporelle et action limitative de trois espèces d'Entomophthoraceae. Entomophaga, 26, 381-393.

Dedryver C.-A., Robert Y., 1977. Quelques problèmes épidémiologiques posés par l'évolution de la répartition verticale de Rhopalosiphum padi, Acyrthosiphon (Metopolophium) dirhodum et Macrosiphum (Sitobion) avenae sur céréales. Ann. Phytopathol., 9, $267-$ 271.

Dransfield R. D., 1979. Aspects of host-parasitoid interactions of two aphid parasitoids, Aphidius urticae (Haliday) and Aphidius uzbekistanicus (Lutzhetski) (Hymenoptera, Aphididae). Ecol. entomol., 4, 307-316.

Evenhuis H. H., 1974. Studies on Cynipidae Alloxystinae. 4. Alloxysta macrophadna (Hartig, 1841) and Alloxysta brassicae (Ashmead, 1887). Entomol. Bericht., 34, 165-168.

Evenhuis H. H., 1976. Studies on Cynipidae Alloxystinae. 5. Alloxysta citripes (Thomson) and Alloxysta ligustri n.sp., with remarks on host specificity in the subfamily. Entomol. Bericht., 36, 140-144.

Evenhuis H. H., 1978. Studies on Cynipidae Alloxystinae. 7. Remarks on Cameron's species and discussion of Phaenoglyphis species with incomplete parapsidal furrow. Entomol. Bericht., 38, 169-175.

Evenhuis H. H., Barbotin F., 1977. Studies on Cynipidae Alloxystinae. 6. Phaenoglyphis villosa (Hartig) and Alloxysta arcuata (Kieffer), Entomol. Bericht., 37, 184-190.

Graham M. W. R. (de), 1969. The Pteromalidae of North Western Europe (Hymenoptera, Chalcidoidea). Bull. br. Mus. nat. Hist. Entomol., suppl., 16, 908 p.

Gutierrez A. P., 1970a. Studies on host selection and host specificity of the aphid hyperparasite Charips victrix (Hymenoptera: Cynipidae). 5. Host selection. Ann. entomol. Soc. Am., 63, 14951498.

Gutierrez A. P., 1970b. Studies on host selection and host specificity of the aphid hyperparasite Charips victrix (Hymenoptera? 
Cynipidae ). 6. Description of sensory structures and a synopsis of host selection and host specificity. Ann. entomol. Soc. Am., 63, 1705-1709.

Jackson H. B., Eikenbary R. D., 1971. Bionomics of Aphelinus asychis (Hymenoptera : Eulophidae), an introduced parasite of the sorghum greenbıg. Ann. entomol. Soc. Am., 64, 81-85.

Jones M. G., Dean G. J., 1975. Observations on cereal aphids and their natural enemies in 1972. Entomol. mon. Mag., 111, 69-77.

Jourdheuil P., 1978. Les degrés de spécificité dans les relations hôte-parasite. Ann. Zool. Ecol. anim., 10, 317-333.

Kalina V., Starý P., 1976. A review of the aphidophagous Aphelinidae (Hym., Chalcidoidea), their distribution and hostrange in Europe. Stud. entomol. forest., 2, 143-170.

Kamijo K., Takada H., 1973. Studies on aphid hyperparasites of Japan. II. Aphid hyperparasites of the Pteromalidae occurring in Japan (Hymenoptera). Ins. Matsum (N.S.), 2, 39-76.

Latteur G., 1973. Etude de la dynamique des populations des pucerons des céréales. Premières données relatives aux organismes aphidiphages en trois localités différentes. Parasitica, 29, 134-151.

Latteur G., Destain J., 1980. Etude de l'action des champignons et des hyménoptères parasites inféodés aux populations de Sitobion avenae $\mathrm{F}$. et de Metopolophium dirhodum Wlk. dans le champ expérimental de Milmort en 1978 et 1979. Bull. O.I.L.B./S.R.O.P., III/4, 11-17.

Mackauer M., 1976a. The sex-ratio in field populations of some aphid parasites. Ann. entomol. Soc. Am., 69, 453-456.

Mackauer M., 1976b. An upper boundary for the sex-ratio in a haplodiploid insect. Can. Entomol., 108, 1399-1402.

Mackauer M., Starý P., 1967. Hym. Ichneumonoidea, world Aphidiidae. In V. Delucchi \& G. Remaudière (Eds.), Index of entomophagous insects, Le François, Paris, 195 p.

Michel M.-F., 1969. Contribution à l'étude des Aphelinidae aphidiphages et de leurs hôtes en France (Hym. Chalcidoidea). Entomophaga, 14, 439-446.

Michel M.-F., 1970. L'adaptation d'Aphelinus asychis Walker (Hym. Aphelinidae) à certains de ses hôtes naturels (Hom. Aphididae). C.R. Acad. Sci., Paris, sér. D, 271, 2339-2341.

Monadjemi M.-N., 1972. Sur les variations de la fécondité d'Aphelinus asychis (Hym. Aphelinidae) en fonction des espèces aphidiennes mises à sa disposition à différentes périodes de sa vie imaginale. Ann. Soc. entomol. Fr. (N.S.), 8, 451-460.

Powell W., 1980. Toxares deltiger (Haliday) (Hymenoptera: Aphidiidae) parasitising the cereal aphid, Metopolophium dirhodum (Walker) (Hemiptera: Aphididae), in southern England: a new host-parasitoid record. Bull. entomol. Res., 70, 407-409.

Rabasse J.-M., 1982. Remarques sur l'identification des Aphidius (Hym., Aphidiidae) parasites des pucerons des céréales. Bull. Soc. entomol. Mulhouse, avril-juin 82, 17-19.

Rabasse J. M., Brunel E., 1977. Cavariella aegopodii Scop. (Hom., Aphididae) en culture de carotte dans l'Ouest de la France. II. Régulation naturelle par Aphidiides (Hym.) et Entomophthorales. Ann. Zool. Ecol. anim., 9, 481-496.
Rabasse J. M., Dedryver C. A., 1982. Overwintering of primary parasites and hyperparasites of cereal aphids in western France. C.R. Réunion groupe d'experts C.C.E., Portici, 23-24 nov. 1982, $11 \mathrm{p}$.

Rabasse J. M., Lafont J. P., Delpuech I., Silvie P., 1982. Progress in aphid control in protected crops. Bull. O.I.L.B./S.R.O.P. (sous presse).

Rabbinge R., Ankersmit G. W., Pak G. A., 1979. Epidemiology and simulation of population development of Sitobion avenae in winter wheat. Neth. J. Plant Pathol., 85, 197-220.

Rautapáa J., 1972. Notes on the bionomics of Aphelinus asychis Walker (Hym. Eulophidae), a parasite of Macrosiphum avenae (F.) (Hom., Aphididae). Ann. Entomol. Fenn., 38, 176-179.

Robert Y., 1979. Recherches écologiques sur les pucerons Aulacorthum solani Kltb., Macrosiphum euphorbiae Thomas et Myzus persicae Sulz. dans l'Ouest de la France. II. Importance du parasitisme par Hyménoptères Aphidiidae et Entomophthora sur pomme de terre. Ann. Zool. Ecol. anim., 11, 371-388.

Robert Y., Rabasse J.-M., 1977. Rôle écologique de Digitalis purpurea dans la limitation naturelle des populations du puceron strié de la pomme de terre Aulacorthum solani par Aphidius urticae dans l'Ouest de la France. Entomophaga, 22, 373-382.

Robert Y., Rabasse J.-M., Rouze-Jouan J., 1972. Régulation naturelle des populations de Capitophorus horni Börner (Hom. Aphididae) par Hyménoptères Aphidiidae et Entomophthorales. Entomophaga, 17, 59-69.

Shalaby F. F., Rabasse J. M., 1979. On the biology of Aphidius matricariae Hal. (Hymenoptera; Aphidiidae), parasite on Myzus persicae Sulz. (Homoptera, Aphididae). Ann. agric. Sci., Moshtohor, 11, 75-97.

Starý P., 1973. A review of the Aphidius species (Hymenoptera, Aphidiidae) of Europe. Annot. zool. bot., Bratislava, 84, 1-85.

Starý P., 1976. Parasite spectrum and relative abundance of parasites of cereal aphids in Czechoslovakia (Hymenoptera, Aphidiidae; Homoptera, Aphidoidea). Acta entomol. bohemoslov., 73, 216-223.

Starý P., 1977. Dendrocerus hyperparasites of aphids in Czechoslovakia (Hymenoptera, Ceraphronoidea). Acta entomol. bohemoslov., 74, 1-9.

Starý P., Remaudière G., Leclant F., 1973. Nouvelles données sur les Aphidiides de France (Hym.). Ann. Soc. entomol. Fr. (N.S.), 9, 309-329.

Sullivan D. J., 1972. Comparative behavior and competition between two aphid hyperparasites: Alloxysta victrix and Asaphes californicus (Hymenoptera: Cynipidae: Pteromalidae). Environ. Entomol., 1, 234-244.

Takada H., 1973. Studies on aphid hyperparasites of Japan. I. Aphid hyperparasites of the genus Dendrocerus Ratzeburg occurring in Japan (Hymenoptera : Ceraphronidae). Insecta matsum., 2, $1-37$ 